\title{
Wearable Device Monitoring Exercise Energy Consumption Based on Internet of Things
}

\author{
Xiaomei Shi ${ }^{1}$ and Zhihua Huang $\mathbb{D}^{2}$ \\ ${ }^{1}$ Guangxi University Xingjian College of Sciences and Liberal Arts, Nanning, Guangxi 530004, China \\ ${ }^{2}$ Department of Sport, Guangxi University, Nanning, Guangxi 530004, China \\ Correspondence should be addressed to Zhihua Huang; huangzhihua@gxu.edu.cn
}

Received 5 September 2020; Revised 2 November 2020; Accepted 21 January 2021; Published 1 February 2021

Academic Editor: Wei Wang

Copyright (c) 2021 Xiaomei Shi and Zhihua Huang. This is an open access article distributed under the Creative Commons Attribution License, which permits unrestricted use, distribution, and reproduction in any medium, provided the original work is properly cited.

\begin{abstract}
Computer technology and related Internet of things technology have penetrated into people's daily life and industrial production; even in competitive sports training and competition, the Internet of things technology has also been a large number of applications. Traditional intelligent wearable devices are mainly used to calculate the steps of athletes or sports enthusiasts, corresponding physical data, and corresponding body indicators. The energy consumption calculated by these indexes is rough and the corresponding error is large. Based on this, this paper will design a wearable device which can accurately calculate and monitor sports energy consumption based on relevant sensors and Internet of things technology. The corresponding core algorithm is the step counting algorithm, which can accurately calculate the relationship between human motion and the corresponding energy consumption and feed back to the intelligent device. In the experiment, the wearable device designed in this paper is compared with the traditional intelligent device. The experiment shows that the wearable device proposed in this paper is more accurate in energy consumption estimation than the traditional device, and its corresponding energy consumption is relatively small.
\end{abstract}

\section{Introduction}

Internet of things technology has brought a lot of innovations to the development of wearable devices, which moves wearable devices gradually towards the direction of miniaturization and intelligence. Traditional intelligent wearable devices are mainly used in the monitoring of people's movement and related conventional body indicators. Such a monitoring method further improves people's quality of life [1-3]. It can effectively realize the daily monitoring of human health and further timely prevent the occurrence of human-related diseases. Traditional intelligent wearable devices mainly include hardware and software parts $[4,5]$. The hardware part mainly depends on the rapid development of semiconductor industry and related Internet technology. The rapid development of hardware promotes the development of the whole wearable system towards miniaturization, low price, and high sensitivity. At the corresponding software level, it is mainly related to the monitoring of sports state, track, athlete's physical condition, and feedback of related possible problems [6, 7]. Although the traditional intelligent wearable devices have been greatly improved in body shape and intelligence, there are still some problems in the calculation of sports energy consumption and the corresponding motion energy monitoring.

For the research on the software and hardware of Internet of things and related intelligent wearable devices, a large number of researchers and related research institutions have carried out research and analysis on it. At the level of human motion recognition, the main research algorithms include four levels: computer vision detection level, inertial sensor level, wireless sensor network level, and corresponding human motion real-time monitoring and analysis level. Intelligent wearable devices were first analyzed based on computer vision, but this kind of method brings too 
much calculation. At the same time, the corresponding image and data processing equipment loss are also large [8-12]; in view of this problem, the relevant researchers in the United States proposed to use a large number of data sensors for action recognition and motion monitoring. The intelligent wearable device based on this design needs a large number of sensors, and its estimation of the amount of motion still has a relatively large error, but this kind of method is in recognition on the level of human motion; the corresponding accuracy rate can reach more than $90 \%$ [13-15]; in terms of the corresponding monitoring technology of human motion energy consumption, the mainstream methods include human body thermal test, indirect human body thermal test, double mark water heat measurement method, and corresponding accelerometer heat measurement method. In essence, only direct thermal measurement method has relatively accurate accuracy, and other methods exist. However, the instrument corresponding to the direct thermal measurement method is relatively expensive, which increases the corresponding cost of the test [16-20]. Different from the direct thermal test method, the relevant American scientists proposed the piezoelectric measurement method, which mainly trains the corresponding body shape characteristics and the corresponding voltage signal when the human body moves, so as to obtain the corresponding linear regression model. Based on this, we further test the energy consumption of athletes during movement and rest, and the corresponding accuracy of this method is higher [21-23].

Based on this, this paper will be based on the shortcomings of traditional intelligent wearable devices, using related sensors and Internet of things technology to design a wearable device that can accurately calculate and monitor the energy consumption of sports. The corresponding core algorithm is the step counting algorithm, which can accurately calculate the relationship between human motion and the corresponding energy consumption and feed back to the intelligent device. In the experiment, the wearable device designed in this paper is compared with the traditional intelligent device. The experimental results show that the system designed in this paper has high accuracy in improving the sports energy consumption.

In this paper, the structure of the article is arranged as follows: the second section mainly analyzes and studies the core algorithm, step counting algorithm, of this paper; the third section focuses on the analysis and research of the software and hardware design of intelligent wearable device; the fourth section of this paper will carry out relevant experiments; finally, the fifth section will summarize.

\section{Research and Analysis of Step Counting Algorithm for Intelligent Wearable Device}

The key algorithm of this system is the realization of step counting algorithm. The main steps of this algorithm are preprocessing of the athlete's motion signal, such as the preprocessing of the athlete's motion estimation and spurious step estimation. The corresponding algorithm processing flow is shown in Figure 1.
In the corresponding data acquisition level, hardware assistance is needed. In this process, the hardware sensors need to be fixed in different body parts of athletes. The corresponding arm signal generally collects the superposition signal of trunk movement and arm movement, and the corresponding waist mainly collects the posture change corresponding to the trunk movement. In the data acquisition level, the simple transmission is used. The sensor device can realize the real-time monitoring of human motion state, so as to reduce the computational complexity of the whole system; at the same time, it will not cause the corresponding storage space to be wasted.

In the corresponding acceleration data processing level, three axes in one algorithm are used to process, which can avoid the inaccurate expression of human body movement signs caused by the corresponding up and down swing of the human body. The corresponding core processing formula is formula (1). In the actual data preprocessing, the normal experiment will collect about 100 data points. The corresponding $\mathrm{A}$ in the corresponding formula represents the corresponding motion data:

$$
A_{i}=\sqrt{A_{x}^{2}+A_{y}^{2}+A_{z}^{2}} .
$$

In the corresponding signal filtering level, the corresponding image and signal noise are filtered out. After the corresponding signal processing, the corresponding signal acceleration processing will be smoother, the corresponding peak value will be more obvious, and the corresponding motion noise burr will be greatly reduced.

The peak detection is mainly used in the identification of the corresponding athlete's movement posture. The corresponding detection algorithm is mainly subtracting the acceleration data and judging based on the corresponding positive and negative values. When the corresponding positive and negative values change, the corresponding peak value is considered to be detected. In the actual detection process, the system sets a threshold value, corresponding to the peak value. If the valley value is less than the corresponding threshold value, the corresponding acquisition data will be filtered out, so as to improve the corresponding accuracy.

In the corresponding level of removing clutter peak, the main problem is to solve the clutter peak of the corresponding peak wave, so as to eliminate the corresponding clutter peak interference, and the corresponding threshold value should be limited in the actual removal of clutter peak. At the level of filtering pseudosteps, it mainly deals with the corresponding small steps and the corresponding actions that interfere with the calculation of motion. In the actual removal of false steps, the main processing is in the counting threshold. In the actual counting, only when the athlete's corresponding pace has a certain rule can the normal count be carried out.

Based on the abovementioned step counting algorithm, the corresponding estimation formula can be further calculated. The corresponding estimation formula is formula (2). The corresponding $E$ represents the corresponding energy consumption, and the corresponding subsequent 


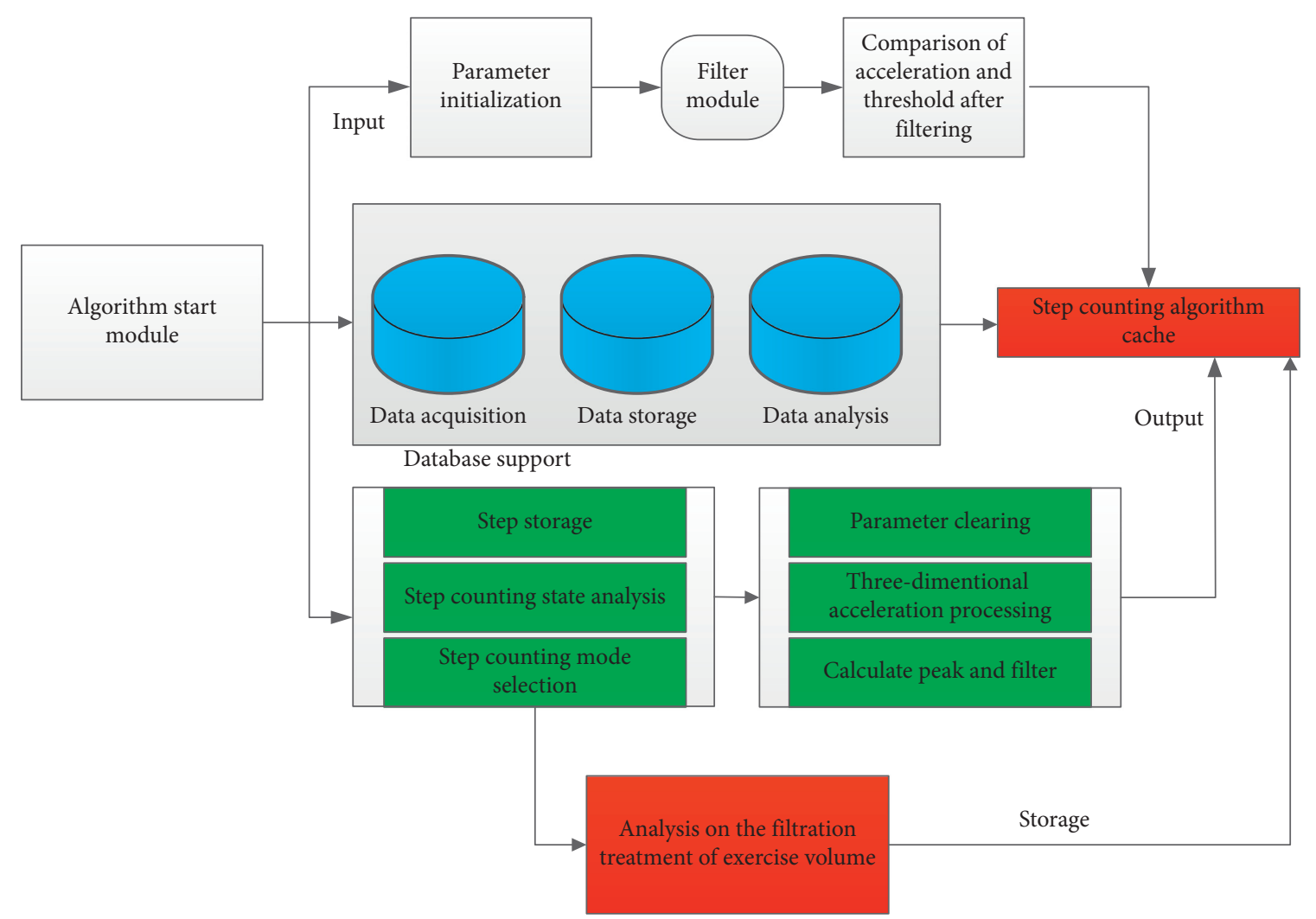

FIGURE 1: Running framework of step counting algorithm in intelligent wearable system.

integral is mainly to integrate the action duration and corresponding action amplitude according to time:

$$
E=\frac{1}{2} \operatorname{umg} \int d a \int t \mathrm{dt} .
$$

The corresponding energy consumption estimation framework of athletes based on step counting algorithm is shown in Figure 2.

Based on the abovementioned step counting algorithm, we can confirm the corresponding athlete's corresponding amount of exercise relatively accurately. At the same time, compared with the traditional algorithm, the step counting algorithm proposed in this section has obvious advantages in algorithm complexity and corresponding algorithm consumption.

\section{Software and Hardware Design of Intelligent Wearable Device}

This section mainly studies and analyzes the software and hardware design of intelligent wearable devices. The corresponding design flow is shown in Figure 3, which includes the software and hardware parts of the whole wearable system. In the hardware design part, it includes the hardware design of the related core modules and the protection circuit of the corresponding part of the module design. In the corresponding software part, it mainly includes the design of the communication part and the related program of the estimation algorithm.
3.1. Hardware Design. The core part of the whole hardware design is the main control and capture part of the wearable device. In the selection of the corresponding processing chip, MSP430 chip of Texas Instruments Company is mainly selected. The corresponding hardware configuration schematic diagram is shown in Figure 4. The corresponding core processor chip needs to be equipped with a $32 \mathrm{kHz}$ crystal oscillator circuit and a $12 \mathrm{Mhz}$ crystal oscillator circuit, and the corresponding power supply setting is $3.3 \mathrm{~V}$. In the actual design, it also needs the corresponding power chip to supply power. From the processing chip, we can see that the system needs more digital to analog converters for the corresponding digital to analog conversion, and each corresponding digital to analog conversion channel needs to reach as many as 22 channels. At the same time, the corresponding Figure 4 also shows the hardware design of the corresponding data acquisition module. At the level of data acquisition module, it needs to collect the attitude information of athletes during the movement. In the selection of sensors, the acceleration sensor and gyroscope are mainly selected to cooperate with each other, the motion attitude sensor and the corresponding inertial sensor, and the corresponding data acquisition needs to use the acceleration transmission. The sensor and gyroscope cooperate so that SPI can realize the mutual transmission with MCU, and the corresponding gyro angular velocity and corresponding dynamic response characteristics can be achieved. In essence, it is necessary to further cooperate with the corresponding acceleration sensor and gyroscope in the data measurement. At the same time, in the corresponding 


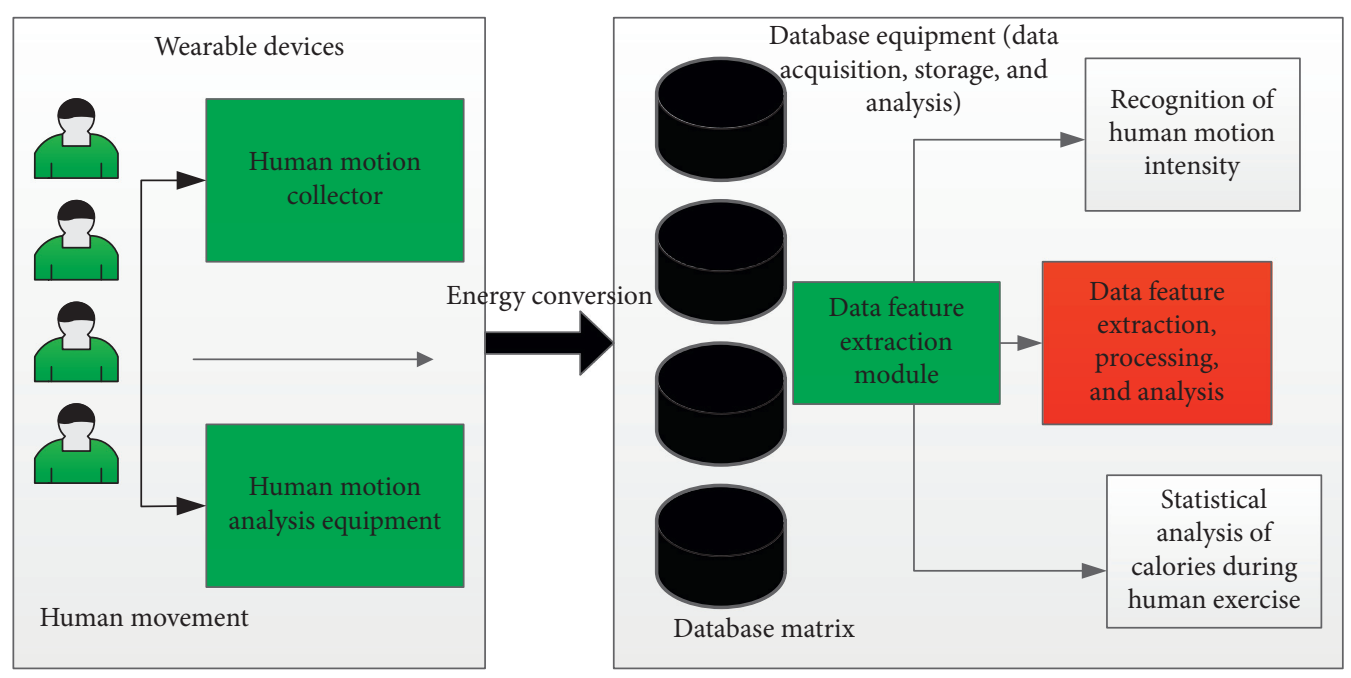

FIgURE 2: Framework of athlete's energy consumption estimation.

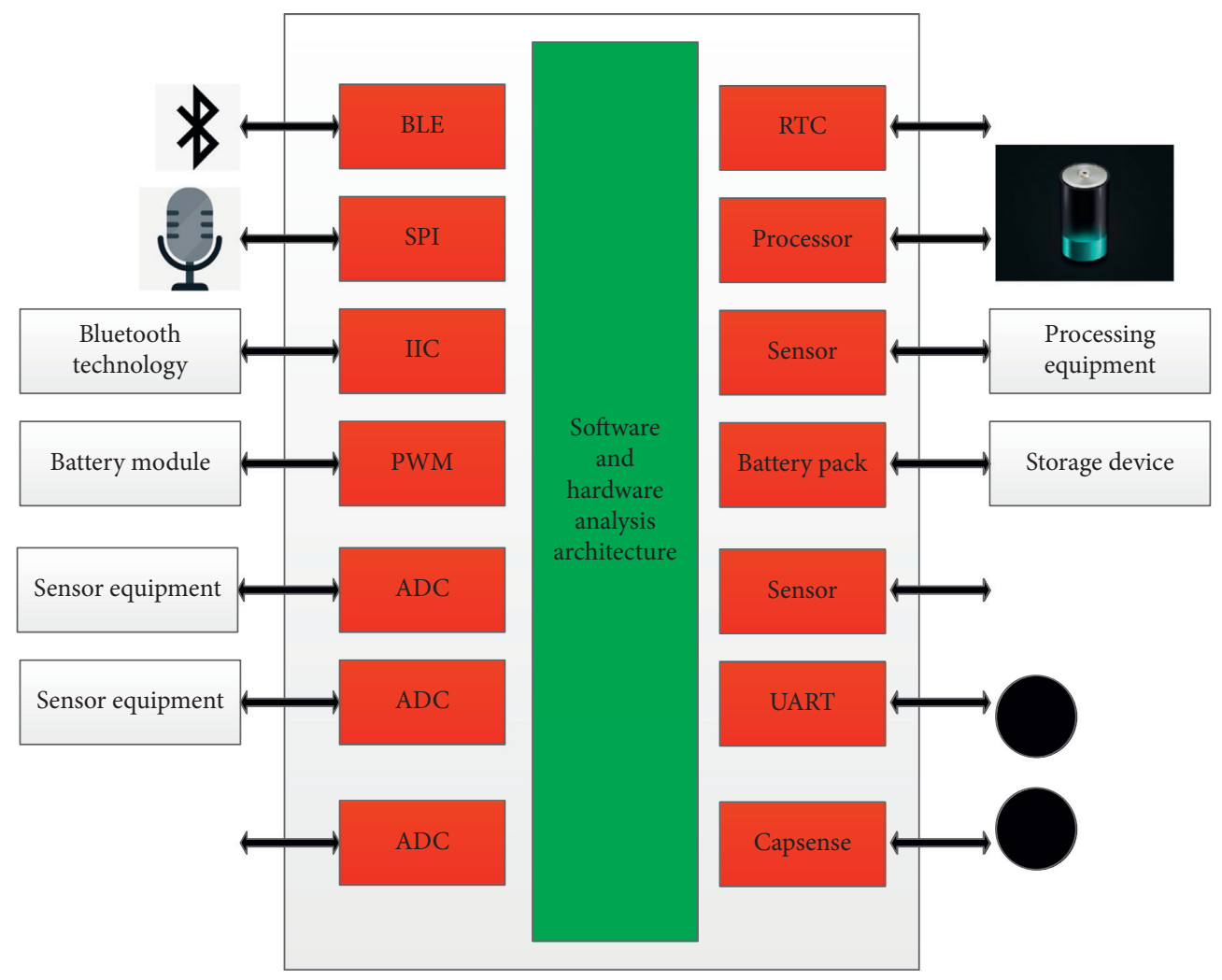

Figure 3: Overall framework of wearable intelligent device.

hardware architecture in Figure 4, it can be seen that the corresponding protection units are designed among different hardware modules, so the protection circuit can realize the safe and reliable operation of the whole system.

Based on the abovementioned hardware acquisition module design, in the hardware data transmission module, this paper mainly adopts Bluetooth module technology. Based on Bluetooth module technology, Bluetooth $2.1+$ EDR data transmission solution is adopted. The data act on data communication, sending related events and at commands through the corresponding UART interface. This hardware structure has the advantages of low energy consumption. When the corresponding module enters idle state or monitoring state, the corresponding module will enter the low power consumption mode.

In the design level of the power module of the corresponding wearable system, the corresponding design block diagram is shown in Figure 5. From Figure 5, it can be seen that the corresponding power module mainly supplies power for all components in the system. The corresponding 


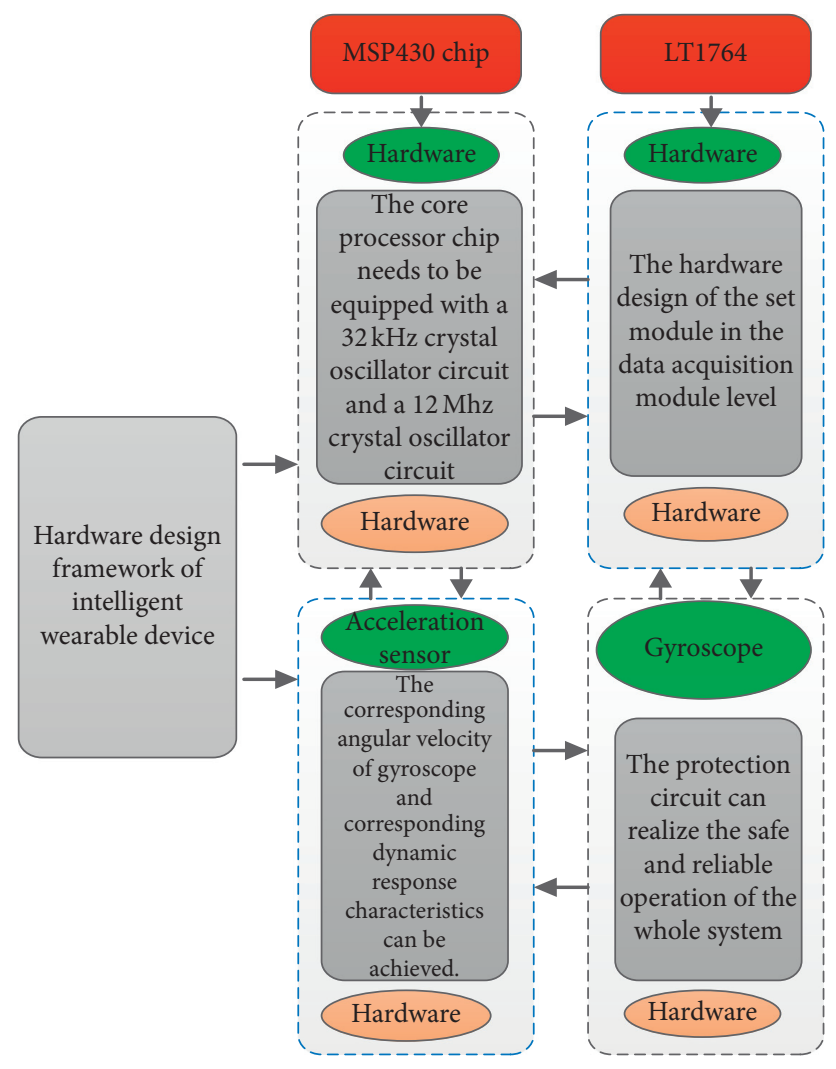

FIGURE 4: Schematic diagram of hardware configuration of wearable intelligent device.

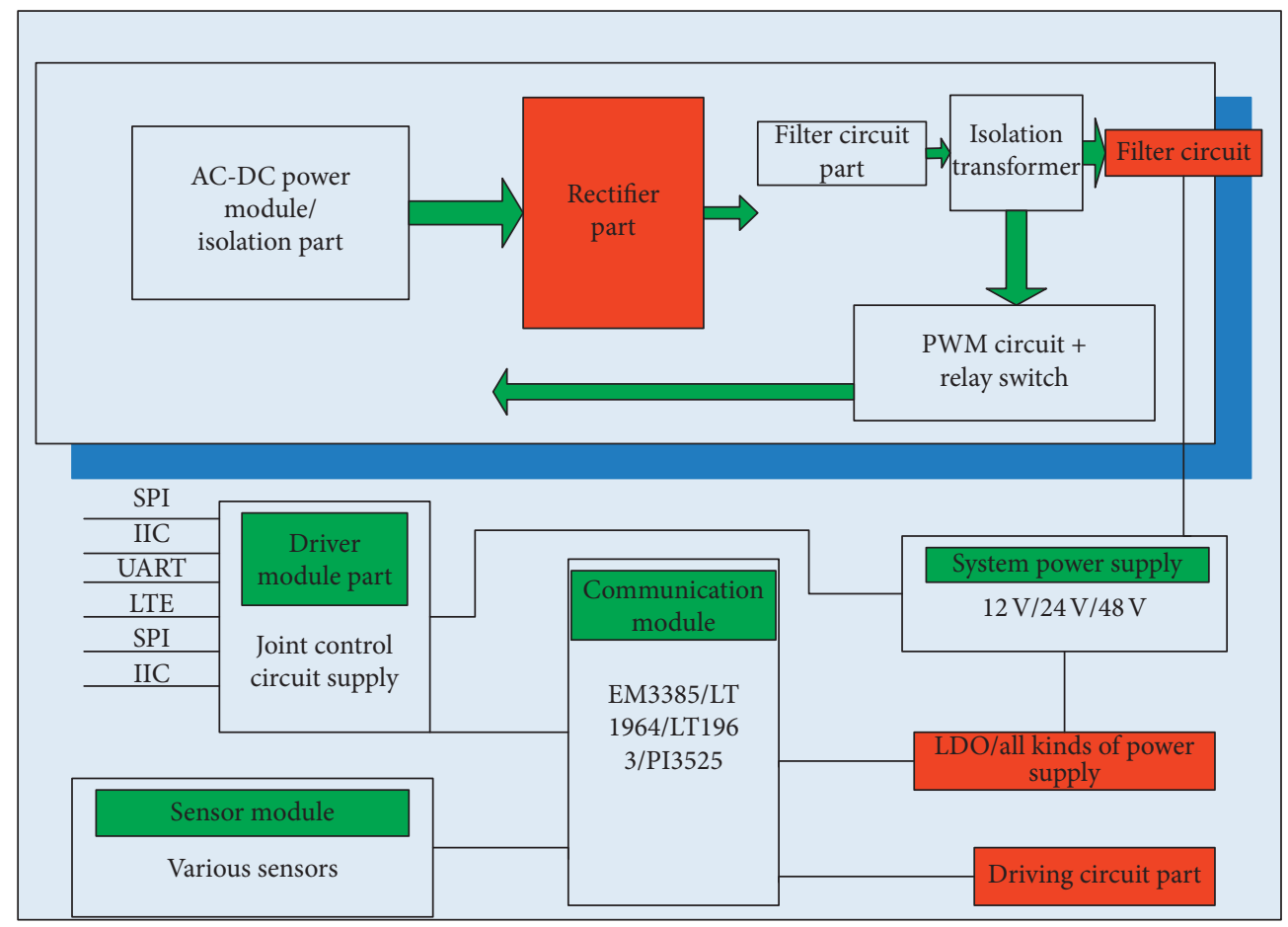

FIgURE 5: Power module block diagram of wearable intelligent device. 


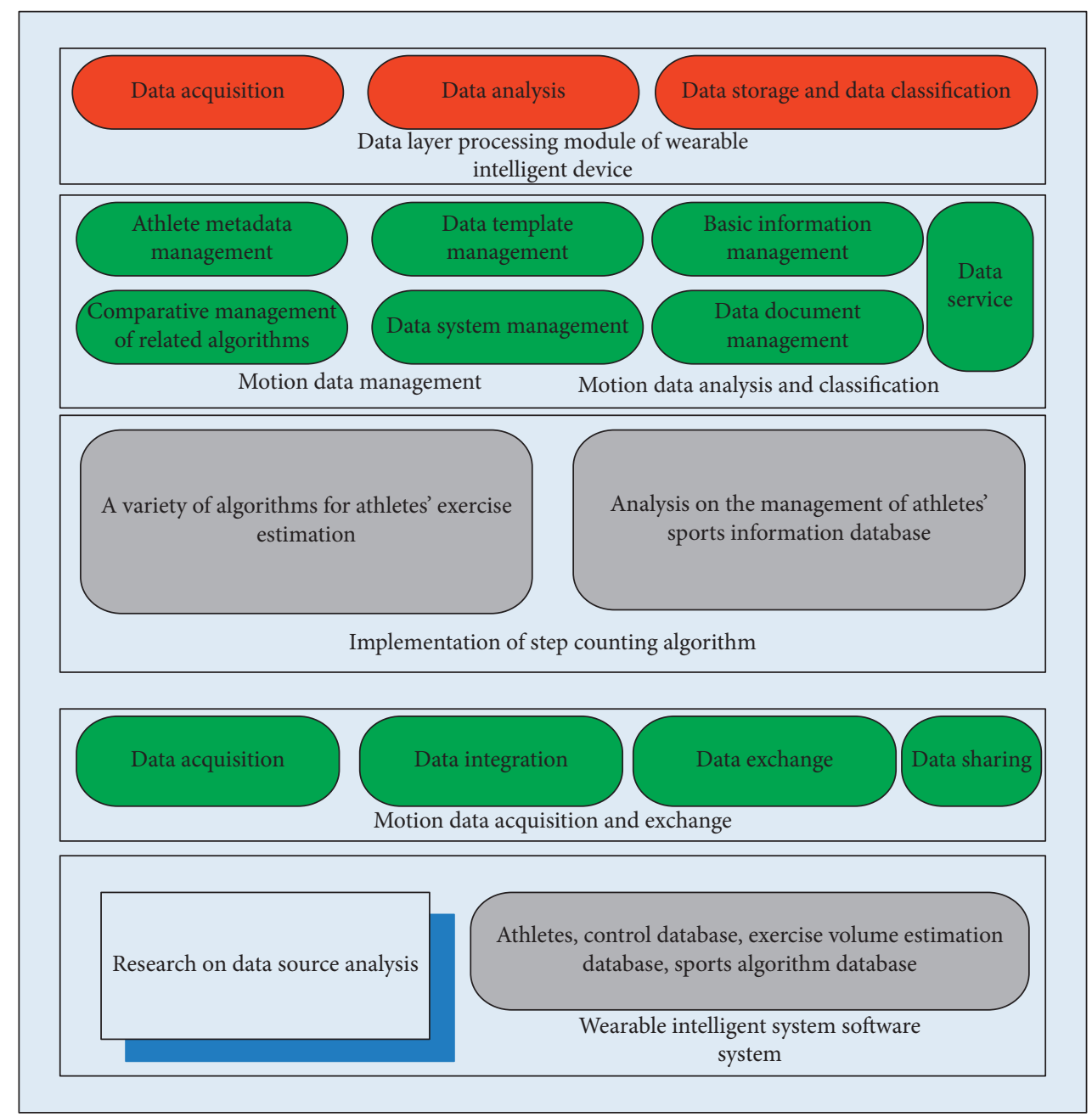

FIGURE 6: Software system block diagram of wearable intelligent device.

mainstream chips mainly select MCP73833 charging management chip and lt1963, lt1964, and other related linear LDO chips. The whole power module is also equipped with corresponding protection circuit; the corresponding main function is to realize the over impact overload protection, charging protection and corresponding under voltage, overcurrent, and other fault conditions.

3.2. Software Design. This paper is mainly based on the embedded development environment of ARM kernel on the corresponding software platform, which mainly includes C compiler, connector, database management layer, and emulator layer. The corresponding software system block diagram is shown in Figure 6. From the figure, we can see that the corresponding software flow is as follows: select a new project, select the corresponding main control chip, add the corresponding chip MDK, enter the project, and write the corresponding source code. In the actual source code writing, pay attention to set the corresponding breakpoints and reasonably configure the corresponding breakpoints, so as to facilitate the later modification and debugging.
In the corresponding communication protocol layer, IIC communication and corresponding SPI communication are mainly selected. In the corresponding IIC communication layer, the power module is mainly connected with MCU, and IIC is transmitted in serial mode as synchronous serial data transmission bus in this system. When the corresponding IIC communication is started, the corresponding data starts to be transmitted, and the host will initiate the signal to carry out the addressing operation through the host. The IIC is used as the bus protocol for data transmission. Each time the data transmitted is 8 bytes. When sending data, the corresponding highest bit needs to add a reply bit for each transmission. There is no limit on the number of words corresponding to the byte in each corresponding communication process. When the transmission is completed, the corresponding host sends a stop signal to end the corresponding communication. In essence, the corresponding MCU is used as the host signal and the corresponding power module is used as the slave part.

In the software design of the corresponding data processing module, the complementary filtering algorithm is mainly used. The corresponding algorithm flow chart of the corresponding 

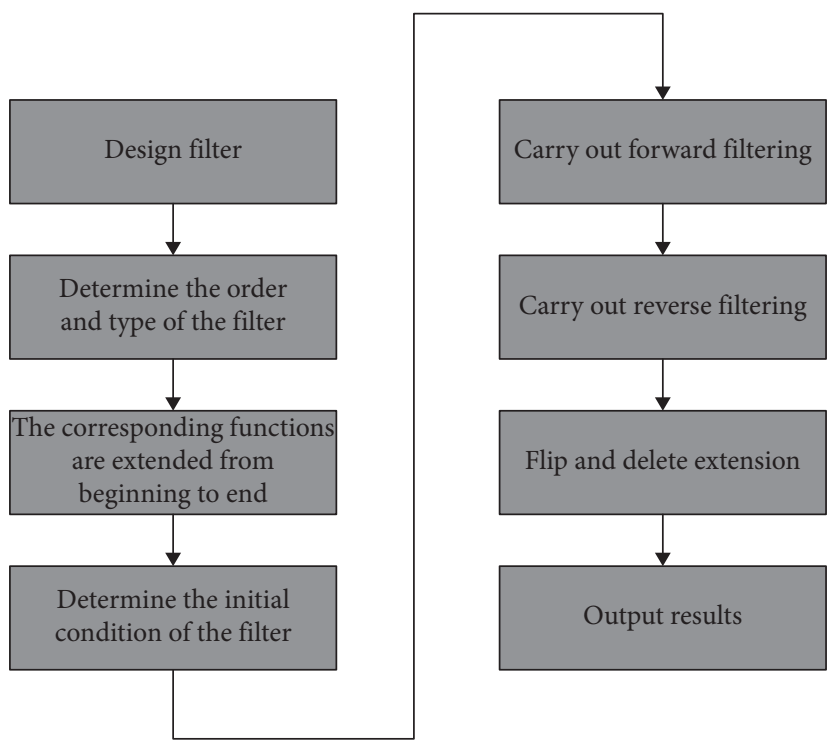

FIGURE 7: Flow chart of complementary filtering algorithm for wearable intelligent devices.

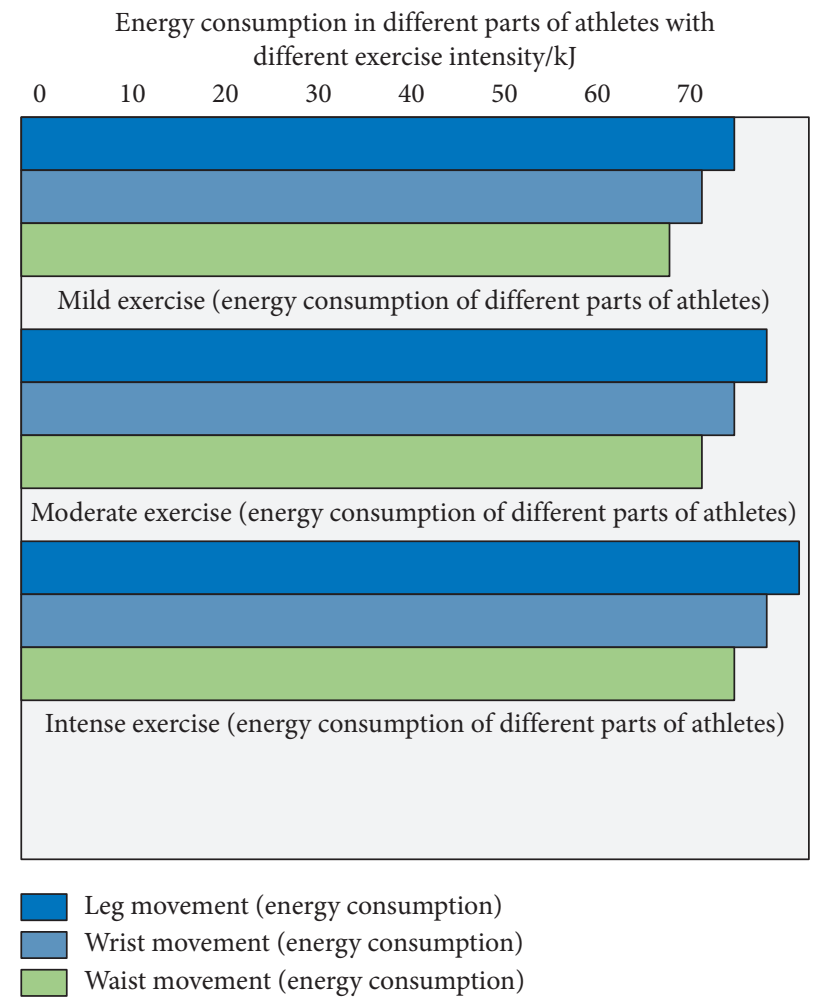

FIGURE 8: Estimation of exercise consumption of different parts of the human body with wearable intelligent device.

complementary filtering is shown in Figure 7. From the figure, we can see that the corresponding solution attitude is as follows: perform initial attitude angle analysis for the corresponding data, read the data from the corresponding sensor, and calculate the corresponding gravity vector.

\section{Experiment Design and Analysis}

In this paper, 20 test objects are selected in the experiment, 19 of them are taken as the corresponding training data and formed a set, and the remaining one is used as the test dataset 


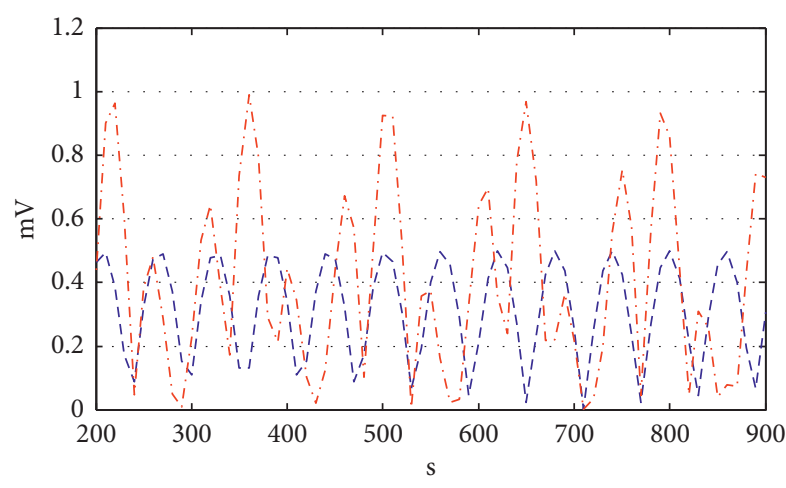

Exercise consumption corresponding to mild exercise

- - - Leg voltage signal curve

...- Arm voltage signal curve

(a)

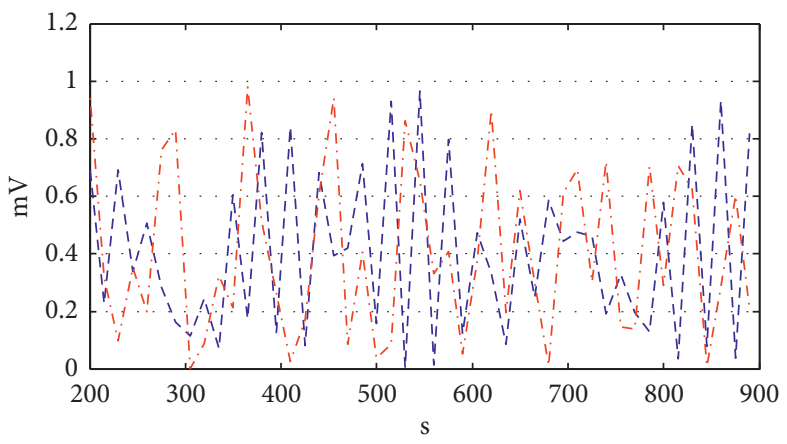

Moderate exercise consumption of different parts of human body

- - - Leg voltage signal curve

-.. Arm voltage signal çbrve

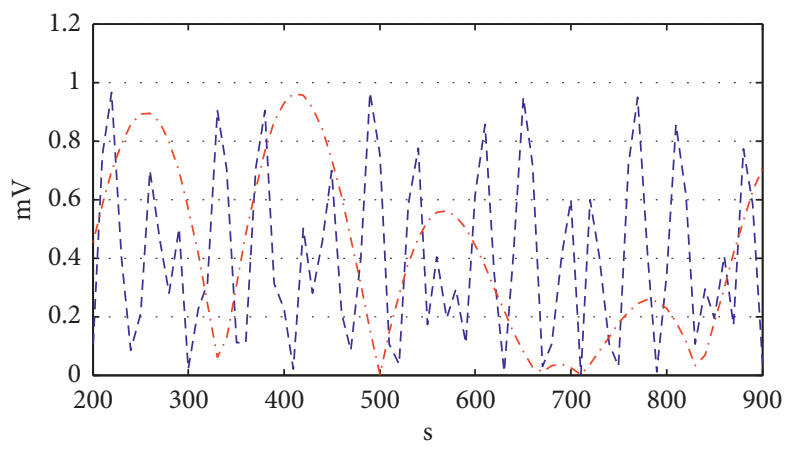

Exercise consumption of different parts

of human body during strenuous exercise

- - - Leg voltage signal curve

. . . Arm voltage signal curve

(c)

Figure 9: Waveform of voltage consumed by different parts of the human body of wearable intelligent device.

for comparative analysis. Based on this experimental group, the corresponding root mean square error and corresponding average absolute error are measured over 20 times. In the analysis of experimental data, the corresponding exercise amount of the tester is mainly analyzed using regression prediction accuracy.

Firstly, the motion intensity recognition of different human body parts of the wearable intelligent device designed in this paper is verified. The corresponding experimental diagram is shown in Figure 8. From the figure, we can see that the corresponding leg is an important part of energy consumption, followed by the corresponding arm part, and finally the corresponding waist. Based on this, the corresponding key parts need to be deployed in the actual sensor layout.
Based on this, the traditional motion estimation algorithm is compared with the estimation algorithm proposed in this paper. When the corresponding experimental samples are moved for the same time, the corresponding energy consumption voltage output waveform is shown in Figure 9, where the corresponding Figure 9(a) represents the corresponding 19 groups of conventional motion consumption, and the corresponding Figure 9(b) represents the corresponding motion based on this paper. The corresponding Figure 9(c) represents the output voltage waveform under the traditional algorithm.

Based on the abovementioned corresponding voltage waveform, we can get the corresponding column chart of athlete's energy consumption estimation in three cases, as shown in Figure 10. From the figure, we can see that the 


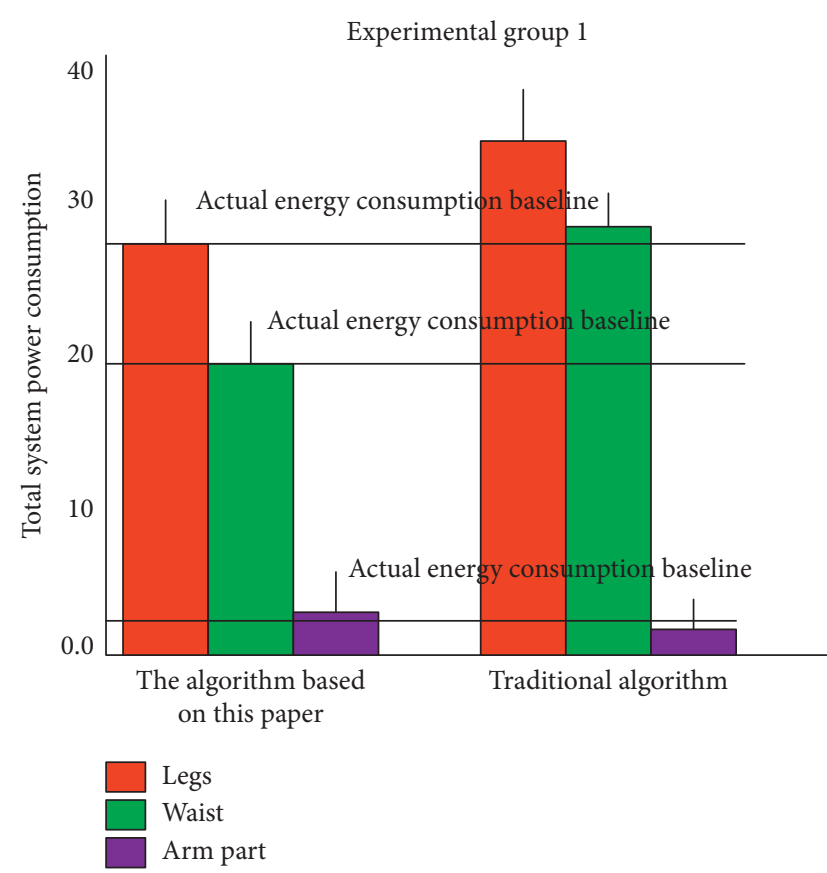

(a)

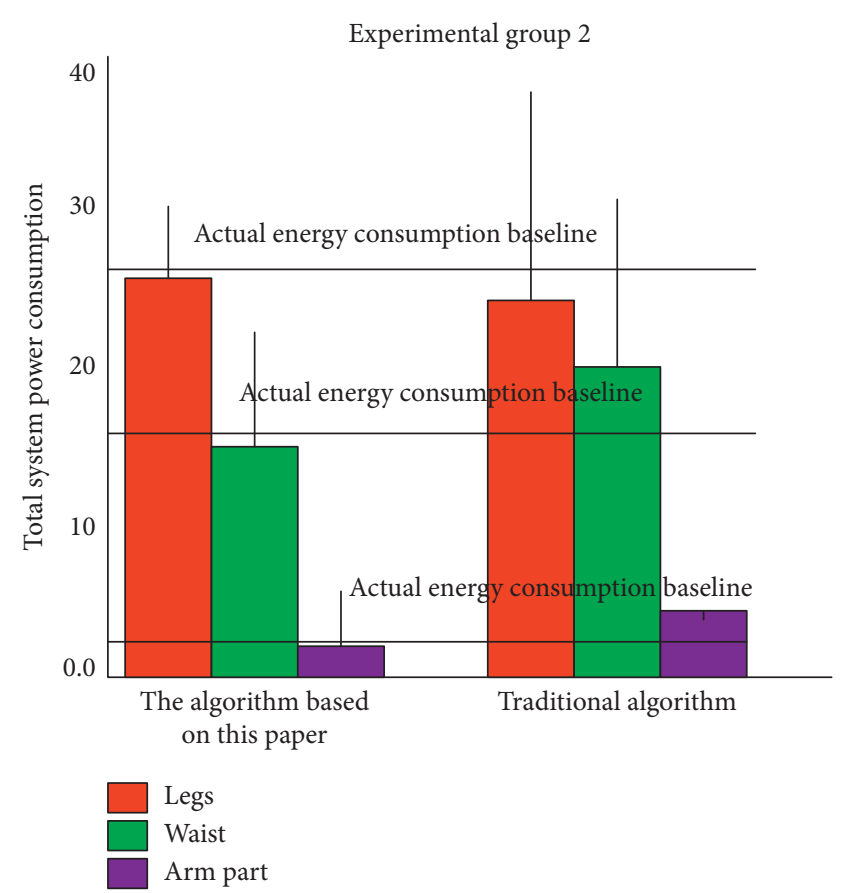

(b)

Figure 10: Column chart of motion energy estimation of different parts of the human body of wearable intelligent device.

algorithm proposed in this paper has obvious advantages, and its corresponding accuracy is higher.

\section{Conclusion}

This paper mainly analyzes and studies the calculation algorithm of athlete's exercise consumption based on the Internet of things technology. Based on the disadvantages of the current intelligent wearable devices, this paper focuses on the analysis and research of a wearable device based on relevant sensors and Internet of things technology, which can accurately calculate and monitor the energy consumption of sports. The corresponding core algorithm is the step algorithm, which can accurately calculate the relationship between human motion and the corresponding energy consumption and feed back to the intelligent device. In the experiment, the wearable device designed in this paper is compared with the traditional intelligent device. The experimental results show that the system designed in this paper has high accuracy in improving the sports energy consumption. This paper will focus on the miniaturization and intelligent application of wearable intelligent devices in the follow-up research and further solve the cost problem. This paper will focus on the application of artificial intelligence algorithm and corresponding neural network algorithm in the corresponding intelligent wearable device, so as to further reduce the energy consumption of the device and improve the accuracy and the corresponding degree of intelligence.

\section{Data Availability}

The data used to support the findings of this study are available from the corresponding author upon request.

\section{Conflicts of Interest}

The authors declare that they have no conflicts of interest or personal relationships that could have appeared to influence the work reported in this paper.

\section{References}

[1] F. Yuling, C. Qingkui, N. N. Xiong et al., "RGCA: a reliable GPU cluster architecture for large-scale internet of things computing based on effective performance-energy optimization," Sensors, vol. 17, no. 8, pp. 1799-1805, 2017.

[2] M. Berthelot, F. P. Henry, J. Hunter et al., "Pervasive wearable device for free tissue transfer monitoring based on advanced data analysis: clinical study report," Journal of Biomedical Optics, vol. 24, no. 6, pp. 1-8, 2019.

[3] A. Martín-Garín, J. A. Millán-García, A. Baïri, J. MillánMedel, and J. M. Sala-Lizarraga, "Environmental monitoring system based on an open source platform and the internet of things for a building energy retrofit," Automation in Construction, vol. 87, no. 8, pp. 201-214, 2018.

[4] C. Xiang and B. Li, "Research on ship intelligent manufacturing data monitoring and quality control system based on industrial internet of things," The International Journal of Advanced Manufacturing Technology, vol. 107, no. 3-4, pp. 983-992, 2020. 
[5] K. Guravaiah and R. Leela Velusamy, "Prototype of home monitoring device using internet of things and river formation dynamics-based multi-hop routing protocol (RFDHM)," IEEE Transactions on Consumer Electronics, vol. 65, no. 3, pp. 329-338, 2019.

[6] L. Xiangju, L. I. Jingzhao, and L. Lina, "Intelligent environment measuring and controlling system of textile workshop based on internet of things," Vascular Pharmacology, vol. 77, no. 5, pp. 80-88, 2015.

[7] Y. Liu, K. Tong, F. Mao, and J. Yang, "Research on digital production technology for traditional manufacturing enterprises based on industrial internet of things in $5 \mathrm{G}$ era," The International Journal of Advanced Manufacturing Technology, vol. 107, no. 3-4, pp. 1101-1114, 2020.

[8] A. Zeeshan and Y. Wonyong, "A survey on energy conserving mechanisms for the internet of things: wireless networking aspects," Sensors, vol. 15, no. 10, pp. 24818-24847, 2015.

[9] C.-F. Lai, S.-Y. Chen, and R.-H. Hwang, "A resilient power fingerprinting selection mechanism of device load recognition for trusted industrial internet of things," IEEE Transactions on Industrial Informatics, vol. 14, no. 8, pp. 3581-3589, 2018.

[10] M. Gao, P. Wang, Y. Wang et al., "Self-powered ZigBee wireless sensor nodes for railway condition monitoring," IEEE Transactions on Intelligent Transportation Systems, vol. 11, no. 2, pp. 900-909, 2018.

[11] G. Berto, M. Luiz, D. S. E. S. Francisco et al., "A middleware with comprehensive quality of context support for the internet of things applications," Sensors, vol. 17, no. 12, p. 2853, 2017.

[12] F. Wang, S. Liu, L. Shu, and X.-M. Tao, "Low-dimensional carbon based sensors and sensing network for wearable health and environmental monitoring," Carbon, vol. 121, no. 4, pp. 353-367, 2017.

[13] B. Zhou and W. Saad, "Minimum age of information in the internet of things with non-uniform status packet sizes," IEEE Transactions on Wireless Communications, vol. 19, no. 3, pp. 1933-1947, 2020.

[14] N.-H. David, T. B. Alejandro, R. T. Javier et al., "Smart vest for respiratory rate monitoring of COPD patients based on noncontact capacitive sensing," Sensors, vol. 18, no. 7, pp. 2144-2156, 2018.

[15] Y. Khan, M. Garg, Q. Gui et al., "Flexible hybrid electronics: direct interfacing of soft and hard electronics for wearable health monitoring," Advanced Functional Materials, vol. 26, no. 47, pp. 8764-8775, 2016.

[16] K. Wang, Y. Wang, Y. Sun, S. Guo, and J. Wu, "Green industrial internet of things architecture: an energy-efficient perspective," IEEE Communications Magazine, vol. 54, no. 12, pp. 48-54, 2016.

[17] M.-F. Francisco, C. G. Pino, and C. G. Cándido, "Authentication based on non-interactive zero-knowledge proofs for the internet of things," Sensors, vol. 16, no. 1, pp. 75-83, 2016.

[18] C. Zhou, H. Luo, W. Fang, R. Wei, and L. Ding, "Cyberphysical-system-based safety monitoring for blind hoisting with the internet of things: a case study," Automation in Construction, vol. 97, no. 2, pp. 138-150, 2019.

[19] M. A. E.-A. Saied, "Health monitoring scheme-based forster resonance energy transfer nanocommunications in the internet of biological nanothings," International Journal of Communication Systems, vol. 33, no. 11, pp. 12-19, 2020.

[20] T.-W. Shyr, J.-W. Shie, C.-H. Jiang, and J.-J. Li, "A textilebased wearable sensing device designed for monitoring the flexion angle of elbow and knee movements," Sensors, vol. 14, no. 3, pp. 4050-4059, 2014.
[21] J.-M. Wang, S. Lan, and W.-K. Li, "Numerical simulation and process optimization of an aluminum holding furnace based on response surface methodology and uniform design," Energy, vol. 72, no. 4, pp. 521-535, 2014.

[22] N. Kasatpibal, N. Viseskul, W. Srikantha, W. Fongkaew, N. Surapagdee, and R. M. Grimes, "Effects of internet-based instruction on HIV-prevention knowledge and practices among men who have sex with men," Nursing \& Health Sciences, vol. 16, no. 4, pp. 514-520, 2014.

[23] M. H. Liang, M. C. M. Couto, C. C. M. Duarte et al., “An internet-based technique for the identification of persons with symptoms of inflammatory polyarthritis of less than 12 weeks," Clinical Rheumatology, vol. 34, no. 3, pp. 465-470, 2015. 